\title{
Distributed Strategies for Channel Allocation and Scheduling in Software-defined Radio Networks
}

\author{
Bo Han*, V. S. Anil Kumar ${ }^{\dagger}$, Madhav V. Marathe ${ }^{\dagger}$, Srinivasan Parthasarathy ${ }^{\ddagger}$, and Aravind Srinivasan ${ }^{\S}$ \\ * Department of Computer Science, University of Maryland, College Park, MD 20742, USA \\ ${ }^{\dagger}$ Department of Computer Science and Virginia Bioinformatics Institute, Virginia Tech, Blacksburg, VA 24061 \\ ${ }_{\ddagger}^{\ddagger}$ IBM T.J. Watson Research Center, Hawthorne, NY 10532 \\ $\S$ Dept. of Computer Science and Institute for Advanced Computer Studies, University of Maryland, College Park, MD 20742
}

\begin{abstract}
Equipping wireless nodes with multiple radios can significantly increase the capacity of wireless networks, by making these radios simultaneously transmit over multiple nonoverlapping channels. However, due to the limited number of radios and available orthogonal channels, designing efficient channel assignment and scheduling algorithms in such networks is a major challenge. In this paper, we present provably-good (centralized and distributed) algorithms for simultaneous channel allocation of individual links and packet-scheduling, in SoftwareDefined Radios (SDR) wireless networks. Our distributed algorithms are very simple to implement, and do not require any coordination even among neighboring nodes. A novel access hash function or random oracle methodology is one of the key drivers of our results. With this access hash function, each radio can know the transmitters' decisions for links in its interference set for each time slot without introducing any extra communication overhead between them. Further, by utilizing the inductivescheduling technique, each radio can also backoff appropriately to avoid collisions. Extensive simulations demonstrate that our bounds are valid in practice.
\end{abstract}

\section{INTRODUCTION}

Significant advances in wireless technology have resulted in cheaper, reliable and adaptive wireless devices. While wireless networks are much easier to deploy in comparison with wireline networks, the phenomenon of wireless interference poses a major challenge in the task of operating a wireless network close to its optimal throughput capacity. One of the techniques used to improve the performance of wireless networks is to design multi-channel multi-radio (MCMR) networks in which each node is equipped with multiple radios that can operate on multiple (non-overlapping) channels. Additionally, recent advances in radio technology have led to the design of SoftwareDefined Radios (SDR) [20], in which packet transmissions on a radio can be switched from one channel to another dynamically. While these advances have lead to an increase in network capacity, they also introduce a host of difficult algorithmic challenges, as nodes need to dynamically make decisions at a per-packet level about which radios and which channels they will employ for communication at any time.

In this work, we deal with a fundamental algorithmic issue that arises in such networks. The problem we address is the following: given a network formed by a set of nodes $V$, and a collection of source-destination pairs $\left(s_{1}, t_{1}\right), \ldots,\left(s_{k}, t_{k}\right)$, what is the maximum throughput capacity of the system, i.e., the maximum rate at which packets can be sent from the sources to their corresponding destinations? ${ }^{1}$ In an SDR network, a node could have multiple radios, each of which could transmit or receive on multiple channels (or called frequency bands in SDR terminology). Wireless interference places constraints on which pairs of radios can communicate simultaneously on the same channel. Under this setting, the throughput optimization presented above decomposes into the following sub-problems: (i) determining the end-to-end throughput $r_{i}$ achieved by connection $i$; (ii) choosing routes for each of the connections $s_{i}$ to $t_{i}$; and (iii) determining which pairs of radios would communicate at each time step and on which channels. Thus, ours is a cross-layer optimization problem involving constraints from the transport (end-to-end rate control), routing, and MAC (channel allocation and link scheduling) layers.

Our central focus in this paper is the joint design and analysis of channel assignment and scheduling strategies. Specifically, given an SDR network, consider the utilization matrix $\mathbf{X}$ whose rows correspond to ordered pairs of radios in the network, columns correspond to channels, and each entry in the matrix specifies the fraction of the time the corresponding pair of radios communicate in the associated channel. The set of all utilization matrices achievable by any channel allocation and link-scheduling scheme is the capacity region of the SDR network. A channel allocation and scheduling scheme $\mathcal{A}$ is $\alpha$-competitive, if for any utilization matrix $\mathbf{X}$ in the capacity region, $\mathcal{A}$ can achieve (component-wise) $\frac{1}{\alpha}$-fraction of $\mathbf{X}$. It is well understood that the competitive ratio of the channel assignment and link scheduling scheme essentially determines the performance ratio of the solution to the overall throughput optimization problem [1]. In fact, as shown in [8], it is also possible to convert an $\alpha$-competitive distributed channel allocation and scheduling scheme into an $\alpha$-competitive distributed strategy for the overall crosslayer optimization problem using distributed network flow mechanisms [5], [2]. Motivated by these observations, we present near-optimal and provably-competitive centralized and

\footnotetext{
${ }^{1}$ Our algorithms in fact solve the more general problem of maximizing $\sum_{i} U_{i}\left(r_{i}\right)$, where $r_{i}$ is the end-to-end throughput achieved by connection $i$ and $U_{i}$ is an arbitrary concave function. The precise functional forms of $U_{i}$ determine the trade-offs between end-to-end throughput and fairness.
} 
distributed schemes for joint channel allocation and scheduling in SDR wireless networks. Our specific contributions are as follows.

\section{OUR CONTRIBUtions}

(a) We present DYNAMIC-SCHEDULER, a centralized algorithm for joint channel assignment and link scheduling and prove that it is $\lambda+2$-competitive. Here, $\lambda$ is the inductive number of the network which is defined as the maximum number of "larger" links which interfere with a given link and which can transmit simultaneously without mutual interference (we define this formally in Section III-C). This parameter is of importance since, for a large class of geometric interference models, the inductive degree of a network is upper bounded by a fixed constant independent of the size or topology of the network [1], [9], [10], [11], [22]. The best known algorithms for link scheduling in single-channel single-radio (SCSR) wireless networks essentially achieve a competitive ratio of $\lambda$ [10], [11], [22]. The significance of DYNAMIC-SCHEDULER arises from the fact that its competitiveness is the best among all known MCMR algorithms proposed thus far in the literature, and is only within an additive factor of 2 from the best known (centralized or distributed) algorithms for SCSR networks.

(b) We present PLDS, a Purely Localized Distributed Scheme for joint channel assignment and link scheduling and prove that it is $(\Delta+2) \cdot e$-competitive. ${ }^{2}$ Here, $\Delta$ is the independence number of the network which is defined as the maximum number of links which interfere with a given link and which can transmit simultaneously without mutual interference. As in the case of the induction number $\lambda$ (see (a) above), for several interference models, this parameter can also be upper bounded by a fixed constant independent of the size or topology of the network [1], [11].

The key innovation which underlies PLDS is the notation of an access hash function; this is a binary function which is parameterized by (i) an ordered pair of radios; (ii) a channel; and (iii) index of the current time slot. The binary outcome of this function (probabilistically) determines whether or not the pair of radios will communicate over the channel during this time slot. A unique property of the access hash function is that it carefully introduces dependencies in the random choices made by the end-points of a link with the aim of increasing their probability of choosing the same channel, while simultaneously minimizing the probability of other conflicting radios choosing the same channel. Access hash functions may be viewed as a non-trivial and sophisticated generalization of random-access scheduling for SDR networks; here, a stochastic process underlies not only arbitrates the accesses to the wireless medium over time, but also yields the channel assignment. In order to appreciate the significance of the access hash function technique, consider the following natural generalization of

\footnotetext{
2, e' denotes the base of natural logarithms.
}

a well-studied random-access protocol for SCSR networks, namely, $p$-persistent MAC [3]: during each time slot, for a given link, each end-point chooses a channel independently at random and then communicates on this channel with some pre-defined probability. Unlike PLDS, this protocol can be shown to have a competitive ratio which is arbitrarily far from optimal.

(c) We present CFDS, a Collision-Free Distributed Scheme which combines the access-hash function methodology of PLDS along with the inductive-scheduling techniques of DYNAMIC-SCHEDULER. CFDS achieves a competitive ratio of $(\lambda+2) \cdot e$, and as its name indicates, it is collision-free (i.e., interfering links never transmit simultaneously) although channel assignment and scheduling decisions are made in a completely distributed manner. The catch in this scheme is as follows: it presupposes a protocol initialization phase during which each link communicates a single value (its utilization) to other links which interfere with it. Despite this additional overhead, we believe that the collision-free property of CFDS renders it particularly attractive for energy-starved scenarios such as sensor networks. In CFDS, nodes can spend a large fraction of their time in sleep mode and only need to wake up during (locally) pre-computed time slots for communication, with the guarantee that transmissions will never be lost due to collisions.

We emphasize that, to the best of our knowledge, PLDS and CFDS are the first purely local-control distributed algorithms for joint channel assignment and scheduling in SDR networks, which do not require any signalling amongst nodes for individual (per-packet) transmissions. A salient aspect of our work is that the competitive factors we derive for all our schemes are independent of the topological properties of the network such as size, degree, the number of radios available at a node, and the number of channels available at a radio; instead, they are fixed constants that depend only upon the specific interference model we assume. Variations in the physicallayer transmission technologies and link-layer schemes have resulted in a large variety of interference models being studied in the literature. Our work presents a unified framework for SDR throughput optimization across these broad spectrum of interference models.

The rest of this paper is organized as follows. We first introduce the background in Section III. In Section IV, we develop necessary conditions for our scheduling problem. We present our centralized and distributed algorithms for joint channel assignment and scheduling in Section V and VI, respectively. In Section VII, we show implications of our algorithms for geometric interference models. The simulation results are summarized in Section VIII. After reviewing related work in Section IX, we conclude in Section X.

\section{BACKGROUND}

We start by presenting our network model, followed by the interference models for SCSR wireless networks and then the models for SDR networks. 


\section{A. Network model}

We use the disk-graph model for the physical layer [1], [10] and model the wireless network as a directed graph $G=(V, E)$, where $V$ denotes the set of nodes in the network and $E$ denotes all ordered pairs of nodes in the network across which direct communication is possible. Each node $v \in V$ is equipped with a collection of radios denoted by $\operatorname{Radios}(v)$; each radio $\rho$ is associated with a collection of non-interfering channels denoted by Channels $(\rho)$. We assume that radios can dynamically switch their channels by using softwaredefined radio technology. Unlike the usual model of MCMR networks where the entire system has a fixed collection of common channels, we make the more general assumption that each radio has its own collection of channels. As mentioned above, for a successful transmission to occur on a link $\ell$, the transmitting and receiving radios of $\ell$ need to use the same channel.

For simplicity, we assume that all the radios at a node $u$ transmit at the same power level, and there is an edge $(u, v)$ if node $v$ can be reached by a transmission from node $u$ at this power level. We will assume a synchronous model of time: time is divided into equi-sized slots that are indexed $0,1,2, \ldots$. We note that this is a common assumption of almost all the existing works for time-slotted scheduling algorithms [1], [8], [22]. We assume that the channel switching latency is negligible compared with the duration of a time slot. An (edge, channel)-pair $(w, \psi)$ has a capacity of $\operatorname{cap}(w, \psi)$ bits/slot. This is the maximum number of bits that can be transmitted across edge $w$ on channel $\psi$ in a single time slot. If $\psi$ is not a channel that is available at both the end-points of edge $w$, then we assume w.l.o.g. that $\operatorname{cap}(w, \psi)$ is zero (since transmitting on channel $\psi$ is not an option for edge $w$ ).

\section{B. Interference in SCSR Networks}

We first describe our interference models in the context of SCSR networks and extend them in Section III-D to SDR networks. We will consider edge-conflict-based interference models which specify interference as a binary relation between pairs of edges in $G$. An interference model specifies, for each edge $w \in E$, a subset of edges $I(w) \subseteq E \backslash\{w\}$. A transmission on edge $w$ during some time slot is successful if and only if no other edges in the set $I(w)$ are active during the same slot. In a single-radio network, each node can be involved in at most one transmission during any time slot. Hence, we will assume w.l.o.g. that all edges other than $w$ that are incident on the end-points of $w$ belong to $I(w)$. In reality, whether two edges in a network interfere or not is determined essentially by their relative locations in space and physical laws of radio propagation. Hence, we will lay special focus on geometric interference models that lay down geometric conditions under which one link interferes with another. Thus, an interference model is not defined w.r.t. a specific network, but is a set of rules for determining conflicting link-pairs in any network. Several geometric interference models have been studied in the literature due to variations in the physical layer hardware of wireless networks as well as differences in physical layer transmission technologies. In all the models below, the nodes are assumed to be embedded in a twodimensional plane. Each node $u$ has a transmission range $r_{t x}(u)$. A necessary condition for edge $w=(u, v) \in E$ to be present is that node $v$ is within a distance of $r_{t x}(u)$ from $u$. We consider seven models:

(a) Node-exclusive model [14]: Only edges that share a common node interfere with each other.

(b) Non-uniform RTS-CTS model [9], [22]: Each node has an interference range, in addition to its transmission range; these ranges can be arbitrary, with the only requirement that the interference range of a node is at least as large as its transmission range. Consider an edge $w=\left(u_{1}, v_{1}\right) \in E$; every edge $w^{\prime}=\left(u_{2}, v_{2}\right)$ such that $u_{1}$ or $v_{1}$ is within the interference range of $u_{2}$ or $v_{2}$ (or vice-versa) is in the interference set $I(w)$.

(c) Uniform RTS-CTS model with parameter $q$ [1]: This is a special-case of the non-uniform RTS-CTS model with the added restriction that all nodes have a uniform transmission range $r_{t x}$, and a uniform interference range $r_{i n t}=q \cdot r_{t x}$, where $q \geq 1$ is a fixed protocol parameter. As in [1], we will consider this model with parameter $q \in\{1,2,2.5\}$; this allows us to obtain stronger performance guarantees than what we show for the non-uniform RTS-CTS model.

(d) Tx-model [10], [25]: Nodes may have non-uniform transmission ranges and interference ranges. Define the interference region of $u$ to be the disk of radius $r_{i n t}(u)$ centered at $u$, where $r_{\text {int }}(u)$ is the interference range of node $u$. Consider the edge $w=\left(u_{1}, v_{1}\right) \in E$; every edge $w^{\prime}=\left(u_{2}, v_{2}\right)$ such that the interference regions of $u_{1}$ and $u_{2}$ overlap in space, is in the interference set $I(w)$. The Tx-model is a simplified form of the RTS-CTS models, as it models interference as a purely transmitter-based phenomenon, while the RTS-CTS models consider interference at both the transmitting and receiving endpoints of an edge. It is also more complex than the uniform RTS-CTS model, since it allows for non-uniform transmission ranges.

(e) fPrIM model [22]: Nodes may have non-uniform transmission ranges and interference ranges; the interference range $r_{\text {int }}(u)$ of each node $u$ is at least a factor $q$ greater than its transmission range $r_{t x}(u)$, where $q>0$ is a fixed constant and a parameter of the model. Consider the edge $w=\left(u_{1}, v_{1}\right) \in E$; every edge $w^{\prime}=\left(u_{2}, v_{2}\right)$ such that the receiver $v_{1}$ is within the interference range $r_{i n t}\left(u_{2}\right)$ of $u_{2}$, is in the interference set $I(w)$.

(f) Protocol model [6], [10]: All nodes have a uniform transmission range $r_{t x}$. Consider the edge $w=\left(u_{1}, v_{1}\right) \in E$; let $\eta>0$ be a fixed constant which is a model parameter. Every edge $w^{\prime}=\left(u_{2}, v_{2}\right)$ such that the transmitter $u_{2}$ is within a distance $(1+\eta) r_{t x}$ of the receiver $v_{1}$, is in the interference set $I(w)$.

(g) $K$-hop model [19]: Define the graph-theoretic distance $d_{\text {hops }}\left(w_{1}, w_{2}\right)$ between two edges $w_{1}$ and $w_{2}$ as follows: $d_{\text {hops }}\left(w_{1}, w_{2}\right)$ is the minimum graph distance (i.e., the number of hops in the shortest hopcount path in $G$ ) between an end-point of $w_{1}$ and an end-point of $w_{2}$. Let $K \geq 1$ be a fixed integer which is a model parameter. Edge $w_{2}$ is 
in the interference set $I\left(w_{1}\right)$ of edge $w_{1}$ if and only if $d_{\text {hops }}\left(w_{1}, w_{2}\right) \leq K$. As in [19], we consider the $K$-hop model on unit disk graphs, where all nodes are assumed to have a uniform transmission range.

In all of the above seven models, if two edges $w_{1}=\left(u_{1}, v_{1}\right)$ and $w_{2}=\left(u_{2}, v_{2}\right)$ share a common end-point, then they are in the interference sets of each other. This captures the constraint that a node can be involved in at most one transmission on any link during a time slot. As is standard convention [17], we define $I_{\text {pri }}\left(w_{1}\right)$ to be the primary interference set of $w_{1}$, which contains all other edges $w_{2}$ that share an end-point with edge $w_{1}$. We also define $I_{s e c}\left(w_{1}\right)=I\left(w_{1}\right) \backslash I_{\text {pri }}\left(w_{1}\right)$ to be the secondary interference set of $w_{1}$; this contains all edges which interfere with $w_{1}$ without sharing any end-point with $w_{1}$.

\section{Independence and Induction in SCSR networks}

We now define the key notions of $\Delta$-independence and $\lambda$ induction for SCSR networks; we then extend them in Section III-D to SDR networks. Given a network $G=(V, E)$ and an associated interference model, we say that the independence number of $G$ is $\Delta(G)$ if $\Delta(G)$ is the maximum number of links that are in the interference set of some specific link $w$, but are mutually interference-free amongst themselves. That is:

$$
\Delta(G) \stackrel{\text { def }}{=} \max _{w \in E} \max _{J \subseteq I(w): \nexists\left(\left(j_{1}, j_{2}\right) \in J \text { s.t. }\left(\left(j_{1} \neq j_{2}\right) \wedge\left(j_{2} \in I\left(j_{1}\right)\right)\right)\right.}|J| .
$$

Given $G=(V, E)$ and an interference model, the induction number of $G$ is $\lambda(G)$ if there exists a total ordering $\succ$ of the network links such that $\lambda(G)$ is the maximum number (taken over all links $w$ ) of links that are (i) $\succ w$, and (ii) in the interference set of link $w$, but are mutually interference-free amongst themselves. Formally, given $\succ$, let $I_{\succ}(w)$ denote (for $w \in E$ ) the set of links that are greater-than $w$ but interfere with $w$. Then,

$$
\lambda(G) \stackrel{\text { def }}{=} \max _{w \in E} \max _{J \subseteq I_{\succ}(w): \exists\left(\left(j_{1}, j_{2}\right) \in J \text { s.t. }\left(\left(j_{1} \neq j_{2}\right) \wedge\left(j_{2} \in I\left(j_{1}\right)\right)\right)\right.}|J| .
$$

Given an interference model and a finite constant $\Delta$, we say that it is $\Delta$-independent if for any network $G=(V, E)$, under this interference model, $G$ 's independence number $\Delta(G)$ is at most $\Delta$. Given an interference model and a finite constant $\lambda$, we say that it is $\lambda$-inductive if for any network $G=(V, E)$, under this interference model, there exists a total ordering $\succ$ of $G$ 's links such that the induction number of $G$ under this ordering is at most $\lambda$. In this case, we will also call the ordering $\succ$ as the greater-than ordering of edges in $E$ which achieves the $\lambda$-induction property. Clearly, while $\Delta(G)$ and $\lambda(G)$ are properties of a given network $G$, the $\Delta$-independence and $\lambda$-induction are properties of an interference model and are independent of the size of the network and the network topology. Also, observe that the requirement of independence is stronger than the notion of induction: an interference model which is $\Delta$-independent is always $\Delta$-inductive. In particular, networks with nodes of heterogeneous (transmission) ranges can have much higher values of $\Delta$ than $\lambda$.
Remark: using $\Delta$ and $\lambda$. The utility of $\Delta$ and $\lambda$ owes to the fact that several geometric interference models considered in the literature have constant induction or constant independence number [1], [9], [10], [11], [22]. For instance, $\lambda$ is at most 5 for the Tx-model, at most 4, 8 and 12 for the Tx-Rx model with parameters 1, 2, and 2.5 [1], [9], [10], [11], [22]. Also, the corresponding ordering $\succ$ of the edges is usually a geometric function: e.g., descending order of the sum of the transmission radii of the two end-points of the edge [9].

\section{Interference and Scheduling in SDR Networks}

It is convenient to employ the notion of an induced radio network, in order to extend our interference models to SDR networks. Given a network $G=(V, E)$, the induced radio network $\mathcal{G}=(\mathcal{V}, \mathcal{L})$ is defined as follows. The set of nodes in $\mathcal{V}$ is the set of all radios which belong to the nodes of $V$. If radio $\rho \in \mathcal{V}$ belongs to the node $u \in V$, then we say that node $u$ is the parent of radio $\rho$. A link $\ell=\left(\rho, \rho^{\prime}\right)$ lies in $\mathcal{L}$ if and only if: (i) the two radios $\rho$ and $\rho^{\prime}$ have at least one channel in common in their channel sets; and (ii) the edge $w=(u, v) \in E$ exists, where $u$ is the parent of $\rho$ and $v$ is the parent of $\rho^{\prime}$. In this case, we will say that edge $w$ is the parent of link $\ell{ }^{3}$ The capacity $\operatorname{cap}(\ell, \psi)$ of a link $\ell \in \mathcal{L}$ when it transmits on channel $\psi$, is equal to $\operatorname{cap}(w, \psi)$, where $w$ is the parent edge of $\ell$.

We now describe a natural extension of our interference models to an induced radio network. If an end-point of link $\ell_{1}$ is the same as an end-point of link $\ell_{2}$ (i.e., they have a radio in common), then they belong to the primary interference sets of each other. For a fixed link $\ell$, we let $\operatorname{Pri}(\ell)$ denote the set of all links that are in the primary interference set of $\ell$. Otherwise, let edges $w_{1} \in E$ and $w_{2} \in E$ denote the parents of links $\ell_{1}$ and $\ell_{2}$; if the end-points of $w_{1}$ and $w_{2}$ have a node in common, then $\ell_{1}$ and $\ell_{2}$ belong to the secondary interference sets of each other. Further, if $w_{2}$ is in the secondary interference set of $w_{1}$, then $\ell_{2}$ is in the secondary interference set of $\ell_{1}$ (and viceversa). We let $\operatorname{Sec}(\ell)$ denote the set of links in the secondary interference set of link $\ell$. The interfering links that share a node in common are called Type I secondary interfering links and the other secondary interfering links are called Type II secondary interfering links. We emphasize that, analogous to the parent network, if two links $\ell_{1}$ and $\ell_{2}$ in the induced radio network interfere with each other, then they cannot both transmit successfully at the same time slot using the same channel. Note that the interference relationship is independent of the frequency of wireless channel used for transmission.

Figure 1 shows an example SDR network with 5 nodes, where each wireless node has two radios. For node $X$, we use $X_{L}\left(X_{R}\right)$ to denote its left (right) radio. In this figure, we only show the active links and ignore the idle links without data transmission. In the following, we use link $\left(A_{R}, B_{L}\right)$ as an example to illustrate the primary and secondary interference set. Based on the definition, this link's primary interference set

\footnotetext{
${ }^{3}$ We will use the terms nodes and edges in the context of the parent graph $G$, and radios and links for $\mathcal{G}$.
} 


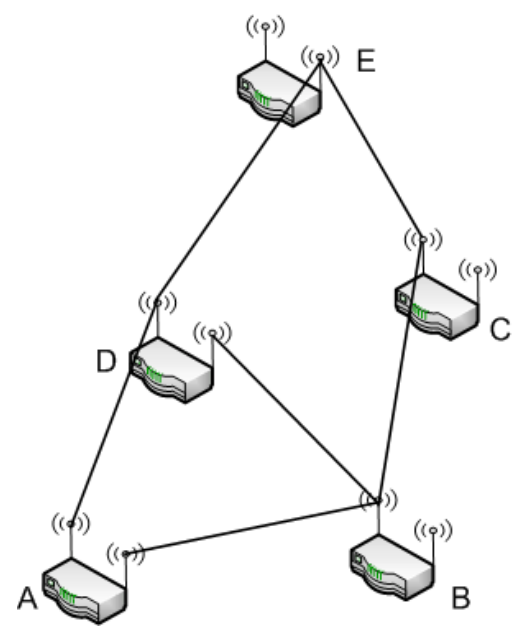

Figure 1. An SDR network with 5 nodes. Each node has 2 radios.

includes two links $\left(B_{L}, C_{L}\right)$ and $\left(B_{L}, D_{R}\right)$, since they share the same radio $B_{L}$ with $\left(A_{R}, B_{L}\right)$. Link $\left(A_{L}, D_{L}\right)$ is in the secondary interference set of link $\left(A_{R}, B_{L}\right)$ because they share a common node $A$. Note that the above relationships have the symmetry property, i.e., if link $\ell_{1}$ is in the interference set of $\ell_{2}$ then $\ell_{2}$ is also in the interference set of $\ell_{1}$. Moreover, the above relationships hold for all the interference models and are independent of the directions of these links. However, for secondary interference, there are also several links introduced by the underlying interference model. Suppose the protocol model is used here and the right radio of node $E$ is out of the interference range of the right radio of node $A$. Link $\left(C_{L}\right.$, $\left.E_{R}\right)$ is also in the secondary interference set of link $\left(A_{R}, B_{L}\right)$ because radio $B_{L}$ (i.e., the receiver node of link $\left(A_{R}, B_{L}\right)$ ) is within the interference range of radio $C_{L}$. However, the symmetry property does not hold for this kind of relationship. Because radio $E_{R}$ is not within the interference range of radio $A_{R}$, link $\left(A_{R}, B_{L}\right)$ is not in the secondary interference set of link $\left(C_{L}, E_{R}\right)$.

Induction and independence in SDR networks: We show how to export these two notions from the parent network to the induced radio network, in Section IV.

Given an induced radio network $\mathcal{G}=(\mathcal{V}, \mathcal{L})$, a schedule $\mathcal{S}$ describes the specific times at which data is moved over the links of the network, and the channel-assignment decisions made for each link during each of its transmissions. In other words, let $Z_{(\ell, \psi), t}$ be the binary scheduling variable which is indexed by the link-channel pair $(\ell, \psi)$ and time slot $t$, and which is defined as follows:

$Z_{(\ell, \psi), t}= \begin{cases}1 & \text { if link } \ell \text { transmits successfully at time } t \text { on } \\ \text { channel } \psi \\ 0 \quad \text { otherwise }\end{cases}$

Let $\Psi$ be the set of all available channels. A schedule $\mathcal{S}$ is an assignment of values to the binary scheduling variables $Z_{(\ell, \psi), t}, \ell \in \mathcal{L}, \psi \in \Psi, t=1,2,3, \ldots$..

Given an induced radio network and a corresponding schedule, we define the utilization value, $x(\ell, \psi)$,

\begin{tabular}{|l|l|}
\hline Notation & Meaning \\
\hline \hline$\rho$ & radio \\
\hline$\ell$ & link \\
\hline $\mathcal{L}$ & the set of links \\
\hline$\psi$ & channel \\
\hline$\Psi$ & the set of all available channels \\
\hline $\mathcal{S}$ & schedule \\
\hline $\mathbf{X}$ & utilization matrix \\
\hline$G$ & directed graph (network) \\
\hline $\mathcal{G}$ & induced radio network \\
\hline$\Delta(G)$ & independence number of $G$ \\
\hline$\lambda(G)$ & induction number of $G$ \\
\hline$I(e)$ & the interference set of edge $e$ \\
\hline Pri $(\ell)$ & the primary interference set of link $\ell$ \\
\hline$S e c(\ell)$ & the secondary interference set of link $\ell$ \\
\hline$H$ & access-hash function \\
\hline$\eta$ & parameter of protocol interference model \\
\hline$q$ & parameter of fPrIM interference model \\
\hline
\end{tabular}

Table I

NOTATION USED IN THIS PAPER.

of each link-channel pair $(\ell, \psi)$ as follows: $x(\ell, \psi) \stackrel{\text { def }}{=}$ $\liminf _{t \rightarrow \infty} \frac{\sum_{j=1}^{t} Z_{(\ell, \psi), j}}{t}$. The utilization $x(\ell, \psi)$ of the pair $(\ell, \psi)$ is the fraction of the time during which link $\ell$ is successfully transmitting on channel $\psi$ in a schedule. A utilization matrix $\mathbf{X}$ specifies the required utilization for each link-channel pair in the network. The rows of $\mathbf{X}$ correspond to the network links, while the columns correspond to channels. The entry $x(\ell, \psi)$ corresponding to row $\ell$ and column $\psi$ denotes the required utilization for the link-channel pair $(\ell, \psi)$. This entry can be non-zero only if both the radios comprising link $\ell$ have $\psi$ as one of their common channels. A utilization matrix is stable if and only if there exists a schedule which meets the utilization requirements for all link-channel pairs, as specified by the matrix. Table I gives a complete list of all the notation used in this paper.

\section{NECESSARY CONDITIONS FOR SCHEDULING}

We now develop two necessary conditions that are satisfied by any stable utilization matrix $\mathbf{X}$. Given a $\lambda$-inductive model and a parent network $G=(V, E)$, we first extend its greaterthan ordering $\succ$ to its induced radio network $\mathcal{G}=(\mathcal{V}, \mathcal{E})$ as follows. Given links $\ell_{1}$ and $\ell_{2}$, if parent $\left(\ell_{1}\right) \succ \operatorname{parent}\left(\ell_{2}\right)$, then we let $\ell_{1} \succ \ell_{2}$. Similarly, if $\operatorname{parent}\left(\ell_{2}\right) \succ \operatorname{parent}\left(\ell_{1}\right)$, we let $\ell_{2} \succ \ell_{1}$. However, if parent $\left(\ell_{1}\right)=\operatorname{parent}\left(\ell_{2}\right)$, then we break the tie between $\ell_{1}$ and $\ell_{2}$ arbitrarily, and order them in some fixed manner. Define $\operatorname{Pri}_{\succ}(\ell)$ as the set of links in $\operatorname{Pri}(\ell)$ that are greater-than $\ell$; define $S e c_{\succ}(\ell)$ analogously. In Theorem 1, we work under an interference model that is $\lambda$-inductive, a parent network $G=(V, E)$, its induced radio network $\mathcal{G}=(\mathcal{V}, \mathcal{L})$, and the greater-than ordering of links in $\mathcal{L}$. In Theorem 2, we work with an interference model that is $\Delta$-independent, and the networks $G$ and $\mathcal{G}$.

Theorem 1: Consider a network $G=(V, E)$ which is $\lambda$ inductive; let $\mathbf{X}$ be a utilization matrix which is defined on $\mathcal{G}$. Matrix $\mathbf{X}$ can be stably scheduled only if the following 
condition holds for all $(\ell, \psi) \in \mathcal{L} \times \Psi$ :

$$
\begin{aligned}
x(\ell, \psi)+ & \sum_{\rho \in \Psi \backslash\{\psi\}} x(\ell, \rho)+\sum_{\chi \in \Psi} \sum_{f \in P r i_{\succ}(\ell)} x(f, \chi) \\
& +\sum_{g \in S e c_{\succ}(\ell)} x(g, \psi) \leq \lambda+2
\end{aligned}
$$

Proof: Consider any valid schedule $\mathcal{S}$; let $Y(\ell, \psi, t)$ be the binary indicator variable which is set to one if link $\ell$ is active on channel $\psi$ at time slot $t$, and is set to zero otherwise. By definition of the $\lambda$-induction property, during any time slot $t$, exactly one of the following two events occur:

1. Link $\ell$ is active on channel $\psi$ and all links in the set $S e c_{\succ}(\ell)$ are inactive on channel $\psi$, or

2. Link $\ell$ is inactive on channel $\psi$ and at most $\lambda$ links in the set $S e c_{\succ}(\ell)$ are active on channel $\psi$.

In either case, we have

$$
\forall(t, \ell, \psi), Y(\ell, \psi, t)+\sum_{g \in S e c_{\succ}(\ell)} Y(g, \psi, t) \leq \lambda
$$

Let $\ell=\left(\rho, \rho^{\prime}\right)$ be the link from radio $\rho$ to radio $\rho^{\prime}$. Each of these radios can support transmission across at most one link at any time. Each link in the set $P r i_{\succ}(\ell)$ is incident on either $\rho$ or $\rho^{\prime}$. Hence, during any time slot $t$, exactly one of the following two events occur:

1. Link $\ell$ is active on some channel in $\Psi$, and all links in the set $P r i_{\succ}(\ell)$ are inactive, or

2. Link $\ell$ is inactive, and at most two links in the set $\operatorname{Pr} i_{\succ}(\ell)$ are active.

In either case, we have $\forall t, \forall(\ell, \psi) \in \mathcal{L} \times \Psi$ :

$Y(\ell, \psi, t)+\sum_{\rho \in \Psi \backslash\{\psi\}} Y(\ell, \rho, t)+\sum_{\chi \in \Psi} \sum_{f \in P r i_{\succ}(\ell)} Y(f, \chi, t) \leq 2$.

By combining (3) and (4), we have $\forall(\ell, \psi, t)$ :

$$
\begin{aligned}
Y(\ell, \psi, t) & +\sum_{\rho \in \Psi \backslash\{\psi\}} Y(\ell, \rho, t) \\
& +\sum_{\chi \in \Psi} \sum_{f \in \operatorname{Pri}_{\succ}(\ell)} Y(f, \chi, t) \\
& +\sum_{g \in \operatorname{Sec}_{\succ}(\ell)} Y(g, \psi, t) \leq \lambda+2
\end{aligned}
$$

The theorem now follows by averaging (5) over all $t$ and by noting that $\lim _{t \rightarrow \infty} \sum_{t^{\prime} \leq t} Y\left(\ell, \psi, t^{\prime}\right) / t=x(\ell, \psi)$.

Theorem 2: Consider a network $G=(V, E)$ which is $\Delta$ independent; let $\mathbf{X}$ be a utilization matrix which is defined on $\mathcal{G}$. Matrix $\mathbf{X}$ can be stably scheduled only if the following condition holds $\forall(\ell, \psi) \in \mathcal{L} \times \Psi$ :

$$
\begin{aligned}
x(\ell, \psi)+ & \sum_{\rho \in \Psi \backslash\{\psi\}} x(\ell, \rho)+\sum_{\chi \in \Psi} \sum_{f \in \operatorname{Pri}(\ell)} x(f, \chi) \\
& +\sum_{g \in \operatorname{Sec}(\ell)} x(g, \psi) \leq \Delta+2
\end{aligned}
$$

The proof is very similar to that of Theorem 1, and is omitted from this version.
Theorem 1 (Theorem 2) essentially states that if the parent network $G$ is $\lambda$-inductive ( $\Delta$-independent), then the induced radio network is $\lambda+2$-inductive $(\Delta+2$-independent $)$. In Sections V and VI, we will use the bounds yielded by Theorems 1 and 2 respectively to establish the performance of our scheduling and channel-assignment algorithms.

\section{Centralized Channel assignment And SCHEDULING}

We now present our centralized algorithm for joint channel assignment and scheduling. We assume that we are given an induced radio network $\mathcal{G}=(\mathcal{V}, \mathcal{L})$ and also a utilization matrix $\mathbf{X}$ defined on $\mathcal{G}$. The basic idea behind our solution (Algorithm 1) is to construct a periodic schedule as follows. Time is divided into frames of $w$ time-slots each; here, $w$ is any large integer such that $w \cdot x(\ell, \psi)$ is integral for all $(\ell, \psi) \in \mathcal{L} \times \Psi$. We compute a conflict-free schedule for a single frame $W$ of size $w$, and repeat it periodically.

The sub-routine which computes a schedule for a single frame is presented in Algorithm 2. Consider the frame $W$ consisting of slots $\{1,2, \ldots, w\}$. For each $(\ell, \psi) \in \mathcal{L} \times \Psi$, the algorithm outputs a conflict-free subset $\operatorname{slots}(\ell, \psi)$; these are the slots at which link $\ell$ transmits on channel $\psi$ within the frame. Initially, for all $(\ell, \psi), \operatorname{slots}(\ell, \psi)$ is empty (Line 2$)$. The algorithm processes the links in $\mathcal{L}$ in the inductive order $\succ$. Suppose the algorithm is processing a specific link $\ell \in \mathcal{L}$; for this link, the algorithm processes each $(\ell, \psi) \in\{\ell\} \times \Psi(\ell)$ in some arbitrarily chosen order where $\Psi(\ell)$ is the set of channels that link $\ell$ can operate on. Consider a pair $(\ell, \psi)$ : the algorithm computes a set conflictSlots $(\ell, \psi)$, which is the set of slots that has already been assigned to any other pair $\left(\ell^{\prime}, \psi^{\prime}\right)$ that conflicts with $(\ell, \psi)$ (Line 8). It then assigns a set of $w \cdot x(\ell, \psi)$ slots to $(\ell, \psi)$ which does not belong to conflictSlots $(\ell, \psi)$ (Line 9). Clearly, Line 9 of Algorithm 2 is not guaranteed to succeed if the utilization matrix $\mathbf{X}$ is not within the capacity region of Algorithm 2. However, Theorem 3 presents sufficient conditions under which $\mathbf{X}$ will be scheduled in a conflict-free manner by Algorithm 2 .

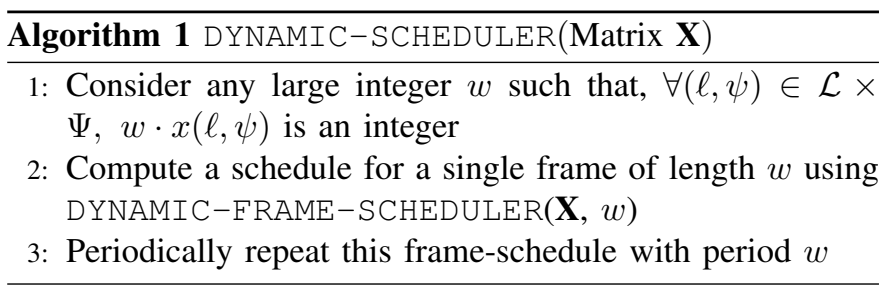

Theorem 3: Consider an induced radio network $\mathcal{G}=(\mathcal{V}, \mathcal{L})$ and its greater-than ordering $\succ$ defined on $\mathcal{L}$; let $\mathbf{X}$ be a utilization matrix which is defined on $\mathcal{G}$. Matrix $\mathbf{X}$ can be stably scheduled by Algorithm 1 if $\mathbf{X}$ satisfies:

$$
\begin{array}{r}
\forall(\ell, \psi), x(\ell, \psi)+\sum_{\rho \in \Psi \backslash\{\psi\}} x(\ell, \rho) \\
+\sum_{\chi \in \Psi} \sum_{f \in \operatorname{Pri}_{\succ}(\ell)} x(f, \chi)
\end{array}
$$




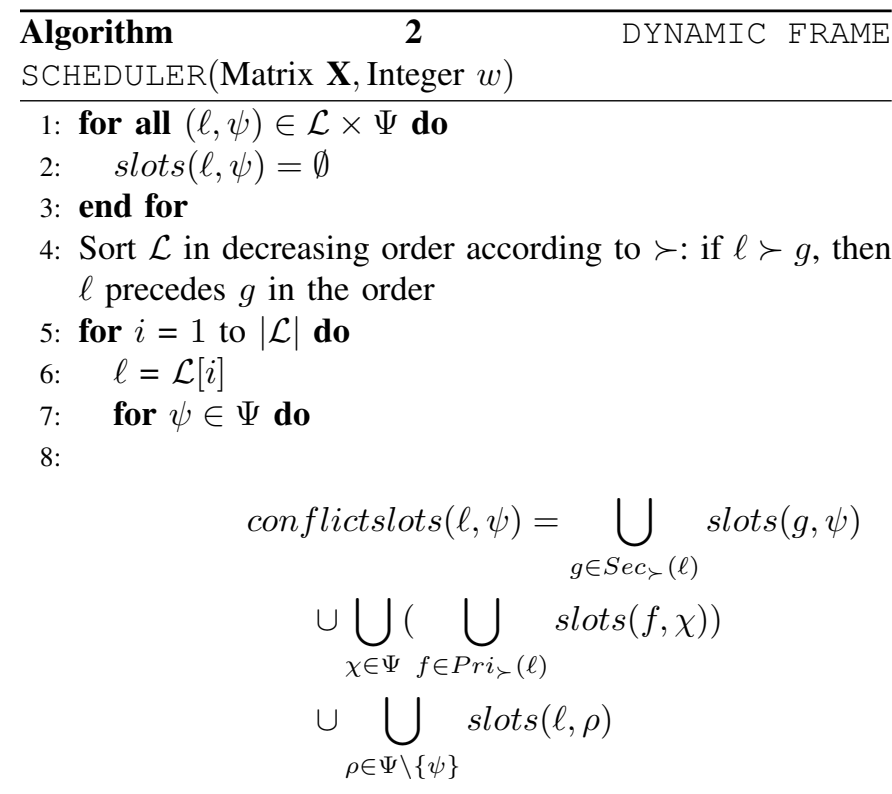

9: $\quad \operatorname{slots}(\ell, \psi)=$ any subset of $W \backslash \operatorname{conflictslots}(\ell)$ of size $w \cdot x(\ell, \psi)$

10: end for

11: end for

Proof: We first note that Line 9 of Algorithm DYNAMIC-FRAME-SCHEDULER is well-defined: i.e., there are always $w \cdot x(\ell, \psi)$ slots available in the set $W$ V conflictslots $(\ell, \psi)$. It follows immediately that the schedule output by Algorithm DYNAMIC-SCHEDULER achieves a utilization of $x(\ell, \psi)$ for each $(\ell, \psi) \in \mathcal{L} \times \Psi$. Assume otherwise: i.e., there exists a pair $(\ell, \psi)$ such that $W \backslash$ conflictslots $(\ell, \psi) \leq w \cdot x(\ell, \psi)$. Hence,

$$
\begin{aligned}
|W|=w< & \mid \text { conflictslots }(\ell, \psi) \mid+w \cdot x(\ell, \psi) \\
\leq & \mid \bigcup_{\rho \in \Psi \backslash\{\psi\}} \operatorname{slots}(\ell, \rho) \\
& \left.\cup \bigcup_{\chi \in \Psi} \bigcup_{f \in \operatorname{Pri}_{\succ}(e)} \operatorname{slots}(f, \chi)\right) \\
& \cup \bigcup_{g \in S e c_{\succ}(e)} \operatorname{slots}(g, \psi) \mid+w \cdot x(\ell, \psi) \\
\leq & \sum_{\rho \in \Psi \backslash\{\psi\}}|\operatorname{slots}(\ell, \rho)| \\
& +\sum_{\chi \in \Psi} \sum_{f \in \operatorname{Pri}_{\succ}(\ell)}|\operatorname{slots}(f, \chi)| \\
& +\sum_{g \in S e c_{\succ}(\ell)}|\operatorname{slots}(g, \psi)|+w \cdot x(\ell, \psi) \\
\leq & \sum_{\rho \in \Psi \backslash\{\psi\}} w \cdot x(\ell, \rho) \\
& +\sum_{\chi \in \Psi} \sum_{f \in \operatorname{Pri}_{\succ}(\ell)} w \cdot x(f, \chi)
\end{aligned}
$$

$$
+\sum_{g \in S e c_{\succ}(\ell)} w \cdot x(g, \psi)+w \cdot x(\ell, \psi)
$$

Dividing by $w$ and rearranging, we get an inequality that

$$
\begin{aligned}
& \forall(\ell, \psi) \in \mathcal{L} \times \Psi, x(\ell, \psi)+\sum_{\rho \in \Psi \backslash\{\psi\}} x(\ell, \rho) \\
& +\sum_{\chi \in \Psi} \sum_{f \in P r i_{\succ}(\ell)} x(f, \chi)+\sum_{g \in S e c_{\succ}(\ell)} x(g, \psi)>1
\end{aligned}
$$

which contradicts (7). This completes the first part of our proof. We next argue that the schedule produced by Algorithm DYNAMIC-SCHEDULER is conflict-free. Consider two pairs $\left(\ell_{1}, \psi_{1}\right)$ and $\left(\ell_{2}, \psi_{2}\right)$ which conflict with each other. W.l.o.g., one of these pairs - say $\left(\ell_{1}, \psi_{1}\right)$ - is considered prior to the other by Algorithm DYNAMIC-FRAME-SCHEDULER in Line 9. Hence, $\operatorname{slots}\left(\ell_{1}, \psi_{1}\right)$ during which the pair $\left(\ell_{1}, \psi_{1}\right)$ is active is a subset of conflictslots $\left(\ell_{2}, \psi_{2}\right)$ and these slots will not be assigned to the pair $\left(\ell_{2}, \psi_{2}\right)$ at all. This proves that the schedule output by Algorithm DYNAMIC-SCHEDULER is a valid conflict-free one.

Competitive Ratio: Comparing (2) and (7), we see that for any feasible matrix $\mathbf{X}$, our centralized protocol can support the matrix $(1 /(\lambda+2)) \mathbf{X}$; thus, the competitiveness of DYNAMIC-SCHEDULER is at most $\lambda+2$.

\section{DISTRIBUTED CHANNEL ASSIGNMENT AND SCHEDULING}

We now present two distributed algorithms for channel assignment and scheduling. The first needs only local information but is not collision-free, and the second completely avoids collisions but needs to exchange interference and linkutilization information during an initial setup phase.

\section{A. Purely Localized Distributed Algorithm}

Our basic approach is as follows. During each slot, each radio in the network chooses at random, one of the links incident on it. It also assigns a channel at random to this link. If the radio is the transmitting end-point of the link, it transmits on the chosen link on the chosen channel. Otherwise, it tries to receive data on the chosen link on the chosen channel. A successful transmission occurs on the link-channel pair $(\ell, \psi)$ during slot $t$, if both the receiving and transmitting end-points of $\ell$ choose link $\ell$, and assign the same channel $\psi$ to $\ell$ at slot $t$, as well as no transmissions are attempted during this slot on other link-channel pairs that interfere with $(\ell, \psi)$. It is clear that if all the radios were to make their choices in a completely uncoordinated manner, each attempted transmission in this scheme will have an abysmally low probability of succeeding; on the other hand, perfect coordination is undesirable and/or expensive in a distributed setting.

The key innovation in our scheme is the use of an accesshash function, which is a parameterized binary hash function that is used by all the nodes in the network, and which carefully introduces dependencies between the various random choices made by the nodes in the network. Such functions are also known as random oracles in cryptography. Before we 
describe its use in our algorithm, we first describes a few key properties of the function. The access-hash function $H$ takes as input three parameters: (i) a link $\ell$, (ii) a channel $\psi$, and (iii) the index of the current time slot $t$. An invocation of $H(\ell, \psi, t)$ returns a value 1 with probability $1-e^{-e \cdot x(\ell, \psi)}$, and a value 0 with probability $e^{-e \cdot x(\ell, \psi)}$. Once the input parameters to $H$ are fixed, the value returned by $H$ does not change. For instance, given that $H(\ell, \psi, t)$ returned 1 when invoked by radio $\rho_{1}$, with probability $1, H(\ell, \psi, t)$ returns value 1 when invoked by another radio $\rho_{2}$. Finally, the random variables $\{H(\ell, \psi, t)\}$ are independent of each other. Popular hashing techniques can be used to construct our access hash function $H$ : we use SHA-1 in our simulations.

We are now ready to describe our distributed algorithm. During slot $t$, a radio $\rho$ computes the values $H(\ell, \psi, t)$ for all the links $\ell$ that are incident on it (i.e., both outgoing and incoming links). It then randomly selects a pair $(\ell, \psi)$ such that $H(\ell, \psi, t)=1$ (if no such pair exists, $u$ sleeps during time $t$ ). If $\ell$ is an outgoing link, it transmits data across $\ell$ on channel $\psi$ during this slot. Otherwise, if $\ell$ is an incoming link, it tunes to channel $\psi$ and awaits an incoming transmission from the transmitting end-point of $\ell$, on channel $\psi$ during this slot. The pseudo-code for the distributed algorithm is presented in Algorithm 3; the actions taken by this algorithm are by a specific radio $\rho$ at a specific time slot $t$ - each radio executes this distributed algorithm during each time slot.

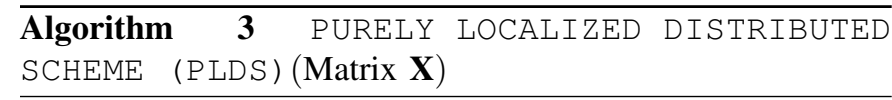

Require: Access hash function $H(\ell, \psi, t)$ such that:

$$
H(\ell, \psi, t)= \begin{cases}1 & \text { with probability } 1-e^{-e \cdot x(\ell, \psi)} \\ 0 & \text { with probability } e^{-e \cdot x(\ell, \psi)}\end{cases}
$$

Require: Given a fixed triplet $(\ell, \psi, t)$, every invocation of $H(\ell, \psi, t)$ yields the same result

Require: The random variables $\{H(\ell, \psi, t)\}$ are independent

1: For all links $\ell \in \mathcal{L}_{\text {out }}(\rho) \bigcup \mathcal{L}_{\text {in }}(\rho)$ (i.e., for all links incident on $\rho$ ), and for all $\psi \in \Psi$, compute $H(\ell, \psi, t)$

2: Randomly pick a pair $(\ell, \psi)$ such that $H(\ell, \psi, t)=1$; if no such pair exists, sleep during time $t$

3: If the selected link $\ell \in \mathcal{L}_{\text {out }}(\rho)$, then schedule an outgoing transmission across $\ell$ on channel $\psi$ at time $t$; else, if $\ell \in$ $\mathcal{L}_{i n}(\rho)$, then tune to channel $\psi$ and await an incoming transmission across $\ell$ on channel $\psi$ at time $t$

We now present a sufficient condition under which Algorithm 3 is guaranteed to achieve the given link utilization matrix $\mathbf{X}$. The stability condition is asymptotic boundedness of queue-sizes as in [14]. Theorem 4 basically shows that the probability of successful transmission on pair $(\ell, \psi)$ at any time $t$ is at least $x(\ell, \psi)$. This theorem and Theorem 5 , technically require an additional slack of $\epsilon$ that can be arbitrarily small but positive: this slack is useful in proving stability by a standard Chernoff-Hoeffding large-deviations approach, which is omitted here for lack of space.
Theorem 4: Let $\epsilon>0$ be an arbitrary constant. Consider an induced radio network $\mathcal{G}=(\mathcal{V}, \mathcal{L})$; let $\mathbf{X}$ be a utilization matrix which is defined on $\mathcal{G}$. Matrix $\mathbf{X}$ can be stably scheduled by Algorithm 3 if

$$
\begin{gathered}
\forall(\ell, \psi), x(\ell, \psi)+\sum_{\rho \in \Psi \backslash\{\psi\}} x(\ell, \rho)+\sum_{\chi \in \Psi} \sum_{f \in \operatorname{Pri}(\ell)} x(f, \chi) \\
+\sum_{g \in \operatorname{Sec}(\ell)} x(g, \psi) \leq \frac{1}{e}-\epsilon
\end{gathered}
$$

Proof: Let $y(\ell, \psi)$ denoted the expected utilization of the pair $(\ell, \psi)$ in the schedule obtained by PLDS: this is the expected fraction of the time link $\ell$ is successfully active on channel $\psi$. We now prove that $\forall(\ell, \psi), y(\ell, \psi) \geq x(\ell, \psi)$, which will suffice. Let $\mathcal{A}(\ell, \psi, t)$ denote the event that link $\ell$ is successfully active on channel $\psi$ during time-slot $t$ (i.e., the pair $(\ell, \psi)$ is chosen for transmission during slot $t$ but no other interfering pair is chosen for transmission during $t$ ). Since the random process which occurs during every time slot is identical, it follows that $y(\ell, \psi)=\operatorname{Pr}[\mathcal{A}(\ell, \psi, t)]$ for an arbitrary fixed $t$. Let $\mathcal{B}(\ell, \psi, t)$ denote the following event: $H(\ell, \psi, t)=1$ and $\forall \rho \in \Psi \backslash\{\psi\}, H(\ell, \rho, t)=0$ and $\forall(f, \chi) \in \operatorname{Pri}(\ell) \times \Psi, H(f, \chi, t)=0$ and $\forall g \in$ $\operatorname{Sec}(\ell), H(g, \psi, t)=0$. Clearly, event $\mathcal{A}(\ell, \psi, t)$ occurs whenever $\mathcal{B}(\ell, \psi, t)$ occurs. Define

$$
\begin{aligned}
E(\ell, \psi) & =\sum_{\rho \in \Psi \backslash\{\psi\}} x(\ell, \rho)+\sum_{\chi \in \Psi} \sum_{f \in \operatorname{Pri}(\ell)} x(f, \chi) \\
& +\sum_{g \in \operatorname{Sec}(\ell)} x(g, \psi)
\end{aligned}
$$

Hence, we have:

$$
\begin{aligned}
y(\ell, \psi)= & \operatorname{Pr}[\mathcal{A}(\ell, \psi, t)] \\
\geq & \operatorname{Pr}[\mathcal{B}(\ell, \psi, t)] \\
= & \operatorname{Pr}[H(\ell, \psi, t)=1] \cdot \Pi_{\rho \in \Psi \backslash\{\psi\}} \operatorname{Pr}[H(\ell, \rho, t)=0] \\
& \Pi_{(f, \chi) \in \operatorname{Pri}(\ell) \times \Psi} \operatorname{Pr}[H(f, \chi, t)=0] \\
& \Pi_{g \in \operatorname{Sec}(\ell)} \operatorname{Pr}[H(g, \psi, t)=0] \\
= & \quad\left(1-e^{-e \cdot x(\ell, \psi)}\right) \Pi_{\rho \in \Psi \backslash\{\psi\}} e^{-e \cdot x(\ell, \rho)} \\
& \quad \Pi_{(f, \chi) \in \operatorname{Pri}(\ell) \times \Psi} e^{-e \cdot x(f, \chi)} \\
& \Pi_{g \in \operatorname{Sec}(\ell)} e^{-e \cdot x(g, \psi)} \\
= & \left(1-e^{-e \cdot x(\ell, \psi)}\right) \cdot e^{-e \cdot E(\ell, \psi)} \\
\geq & \left(1-e^{-e \cdot x(\ell, \psi)}\right) \cdot e^{-e \cdot\left(\frac{1}{e}-x(\ell, \psi)\right) \quad(\text { from }(9))} \\
= & \left(1-e^{-e \cdot x(\ell, \psi)}\right) \cdot e^{e \cdot x(\ell, \psi)-1} \\
= & \left(e^{e \cdot x(\ell, \psi)-1}-e^{-1}\right) \\
= & \frac{e^{e \cdot x(\ell, \psi)}-1}{e},
\end{aligned}
$$

which is at least $x(\ell, \psi)$.

As mentioned prior to the statement of the theorem, the slack of $\epsilon$ is useful in proving stability, which we will demonstrate in the full version of this paper. 


\section{B. Collision-free Distributed Scheduling Algorithm}

The PLDS Algorithm (3) is very simple to implement, i.e., each radio can make its own transmission decision locally. However, it does not guarantee that there are no collisions due to simultaneous transmissions of interfering links. By utilizing the inductive-scheduling technique of Kumar et al. [10], we further improve the PLDS algorithm and develop a collisionfree distributed scheme (CFDS) which is presented in Algorithm 4.

PLDS (Algorithm 3) can not avoid collisions because in the second step of PLDS, each radio makes its transmission decision randomly, without any coordination with other radios. To avoid collisions, the transmitter radio $\rho$ of link $\ell$ needs to know the final transmission decisions for the transmitter radios of $\ell$ 's interfering links. In CFDS, each link exchanges its utilization value with its interfering links during the protocol initialization phase. Therefore, the transmitter radio of link $\ell$ knows the value of $H\left(\ell^{\prime}, \psi, t\right)$ for all $\ell^{\prime} \in I(\ell)$ based on their utilization value $x\left(\ell^{\prime}, \psi\right)$. We also require that for all links $\ell \in \mathcal{L}_{\text {out }}(\rho) \bigcup \mathcal{L}_{\text {in }}(\rho)$ (i.e., for all links incident on $\rho$ ), and for all $\psi \in \Psi$, after computing $H(\ell, \psi, t)$, radio $\rho$ will pick a pair $(\ell, \psi)$ such that $H(\ell, \psi, t)=1$ using SHA1 as follows. The input of SHA-1 is $H(\ell, \psi, t)$ for all links incident on $\rho$ and for all $\psi \in \Psi$. Suppose among these linkchannel pairs, there are $n$ pairs with $H(\ell, \psi, t)=1$. The output of SHA-1 is $x$ and the maximal output of SHA- 1 is $y$. Let $k=\left\lceil\frac{n \times(x+1)}{y+1}\right\rceil$. Radio $\rho$ will choose the $k$ th $(1 \leq k \leq n)$ linkchannel pair with $H(\ell, \psi, t)=1$. Note that for the selected link $\ell$, radio $\rho$ can also know the final transmission decisions for the transmitter radios of $\ell$ 's interfering links using the same procedure, because link $\ell$ knows the utilization value of its interfering links during the protocol setup. After that, $\rho$ can make its proper transmission-decision using the inductivescheduling technique as described in Algorithm 4.

Using the inductive-scheduling technique blindly may not completely avoid collision, due to the asymmetric nature of Type II secondary interfering links. For example, suppose link $\ell_{1}$ is in the secondary interference set of link $\ell_{2}$ but $\ell_{2}$ is not in the secondary interference set of $\ell_{1}$ and $\ell_{2}$ has higher order than $\ell_{1}$. Collision may occur if both of them transmit. In this case, we force $\ell_{2}$ to backoff, no matter what its inductive order is (compared with its Type II secondary interfering links). Theorem 5 provides a sufficient condition under which Algorithm 4 is guaranteed to achieve the given link utilization matrix $\mathbf{X}$. Its proof is very similar to that of Theorem 4, and is omitted from this version.

Theorem 5: Let $\epsilon>0$ be an arbitrary constant. Consider an induced radio network $\mathcal{G}=(\mathcal{V}, \mathcal{L})$ and its greater-than ordering $\succ$ defined on $\mathcal{L}$; let $\mathbf{X}$ be a utilization matrix which is defined on $\mathcal{G}$. Matrix $\mathbf{X}$ can be stably scheduled by Algorithm 4 if $\mathbf{X}$ satisfies:

$$
\begin{array}{r}
\forall(\ell, \psi), x(\ell, \psi)+\sum_{\rho \in \Psi \backslash\{\psi\}} x(\ell, \rho) \\
+\sum_{\chi \in \Psi} \sum_{f \in \operatorname{Pri}_{\succ}(\ell)} x(f, \chi)
\end{array}
$$

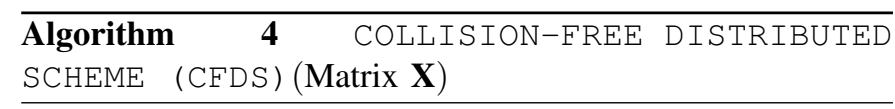

Require: Each link communicates its utilization value to its interfering links during the protocol initialization phase

Require: Access hash function $H(\ell, \psi, t)$ defined in (8)

Require: Given a fixed triplet $(\ell, \psi, t)$, every invocation of $H(\ell, \psi, t)$ yields the same result

Require: The random variables $\{H(\ell, \psi, t)\}$ are independent

1: For all links $\ell \in \mathcal{L}_{\text {out }}(\rho) \bigcup \mathcal{L}_{\text {in }}(\rho)$, and for all $\psi \in \Psi$, compute $H(\ell, \psi, t)$

2: Pick a pair $(\ell, \psi)$ such that $H(\ell, \psi, t)=1$ using SHA1 , based on the value of $H(\ell, \psi, t)$ for all links $\ell \in$ $\mathcal{L}_{\text {out }}(\rho) \bigcup \mathcal{L}_{\text {in }}(\rho)$ and for all $\psi \in \Psi$; if no such pair exists, sleep during time $t$

3: If the selected link $\ell \in \mathcal{L}_{i n}(\rho)$, then tune to channel $\psi$ and await an incoming transmission across $\ell$ on channel $\psi$ at time $t$

4: Else schedule an outgoing transmission across $\ell$ on channel $\psi$ at time $t$, if $\ell \in \mathcal{L}_{\text {out }}(\rho)$ and there are no interfering links with higher inductive order which also decide to schedule a transmission on channel $\psi$

$$
+\sum_{g \in \operatorname{Sec} \succ(\ell)} x(g, \psi) \leq \frac{1}{e}-\epsilon
$$

\section{IMPLICATIONS FOR GEOMETRIC INTERFERENCE MODELS}

Recall that the geometric interference models which we introduced in Section III-B have either bounded-independence or bounded-induction. This fact, along with Theorems 3 and 4 yields the following constant-factor performance guarantees for our scheduling algorithms (recall some sample values for $\Delta$ and $\lambda$ from Section III-C, and see the argument following the proof of Theorem 3 on how to get guarantees such as those of Corollary 6):

Corollary 6: Given any interference model that is $\lambda$ inductive, Algorithm DYNAMIC-SCHEDULER achieves a $\lambda+$ 2 competitive ratio for joint channel assignment and link scheduling. Specifically, when the interference model is the $T x-$ model, the $T x-R x$ model with parameters 1,2 , and 2.5 , the $K$-hop model on unit disk graphs, the geometric RTS/CTS model, the fPrIM model, and the protocol model, algorithm DYNAMIC-SCHEDULER yields a 7 -factor, 6 -factor, 10-factor, 14-factor, 51-factor, $O(1)$-factor, $O(1)$-factor, and $O(1)$-factor competitive ratio respectively for joint channel assignment and link scheduling for multi-radio multi-channel wireless networks.

Corollary 7: Let $\epsilon>0$ be an arbitrary constant. Given any interference model that is $\Delta$-independent, Algorithm PLDS yields a $(\Delta+2) \cdot(e+\epsilon)$-competitive ratio for joint channel assignment and link scheduling. Specifically, when the interference model is the uniform $T x-$ model, the $T x-R x$ model with parameters 1,2 , and 2.5 , the $K$-hop model on unit disk graphs, and the $K$-hop model on $(r, s)$-civilized graphs, algorithm PLDS yields a $7(e+\epsilon)$-factor, $6(e+\epsilon)$ - 
factor, $10(e+\epsilon)$-factor, $14(e+\epsilon)$-factor, $51(e+\epsilon)$-factor, $O\left(\frac{r^{2}}{s^{2}}\right)$-factor competitive ratio respectively for the end-to-end utility maximization problem with multiple radios and multiple channels.

Due to limited space, we omit the detailed proof of these corollaries, but refer interested readers to [10] for a similar proof.

\section{ViII. Simulation Results}

In this section, we show the feasibility of the proposed distributed and localized scheduling algorithm and evaluate its performance through extensive simulations on both regular grids and random networks. A custom simulator developed in $C$ is used for performance evaluation. There are two main goals of our simulations: (1) study the feasibility of our randomized algorithm using SHA-1 as the access hash function, under node-exclusive interference model [14], protocol interference model [6], [10] and fPrIM interference model [22]; and (2) compare the probability of events $\mathcal{A}(\ell, \psi, t)$ and $\mathcal{B}(\ell, \psi, t)$ with $x(\ell, \psi)$ for all the link-channel pairs, under the above three interference models.

\section{A. Network Settings and Simulation Setup}

In the simulation, we assume that each node has two radios as this is the typical setting for most of current IEEE 802.11 SDR mesh network deployments. We also assume that there are 3 available independent channels in the system as it is the case for IEEE $802.11 \mathrm{~b} / \mathrm{g}$ networks (i.e., channels 1,6 and 11). Note that under this setting, there are totally 24 available link-channel pairs in the induced radio network for an edge $(u, v)$, i.e., 4 links from node $u$ to $v$ and verse vice and each link can operate on 3 different channels. For the regular grids, we performance simulations on both $4 \times 4$ and $5 \times 5$ grids. For the random networks, we randomly generate $n$ wireless nodes uniformly in a $500 \times 500$ units region. The transmission range for all the nodes is 100 units in node-exclusive model and protocol model; in the PrIM model, the transmission range for each node is randomly chosen from the set $\{60,80,100$, $120,140\}$ units.

For each interference model, we perform simulations on random networks with 20,50 and 100 nodes. For the case of the fPrIM interference model, the node placements are similar but the transmission ranges of nodes in the networks are nonuniform. Given a sample network, we first randomly generate its utilization matrix $\mathbf{X}$ which satisfies (9) for all the link-channel pairs and then run the simulation for $T$ time slots. To make the randomly generated utilization matrix $\mathbf{X}$ more realistic, we randomly choose $m \%$ of link-channel pairs in $\mathbf{X}$ and offer no traffic load on them. Note that in practice the utilization matrix is essentially determined by routing algorithms and our scheduling algorithm does not rely on any particular routing algorithms. Therefore, here we use the randomly generated utilization matrix for simplicity.

The popular hash function SHA-1 is a good access hash function and is used as the access-hash function $H$ in our current simulation. Suppose all the probabilities can be approximated very well by rationals of the form $a / 2^{b}$, where $2^{b}$ is some large integer. Then, we choose a random subset $R$ of size $b$ from SHA-1's 160-bit output and compare the new integer $c$ formed by these bits in $R$ with $a=2^{b}\left(1-e^{-e \cdot x(\ell, \psi)}\right)$ to determine the value of $H(\ell, \psi, t)$ (i.e., $c<a$ means $H(\ell, \psi, t)=1)$. In our simulation, $R$ is pre-distributed to all nodes and we take $b=40$.

In the following subsections, we first give some interference model dependent parameters and then present the simulation results for each interference model. For the randomly generated utilization matrix, we report the range (i.e, the min and max values) and average values of the sum in the LHS of (9) for all the link-channel pairs. During the generation of the utilization matrix, we try to make the maximal sum as close to $1 / e$ as possible. The reason is that if the sums are much smaller than $1 / e$, which means the traffic loads are low and thus the contention probabilities are low, we do expect good performance of the proposed randomized algorithm. For each interference model, we report the worst case (minimal) ratios between the probabilities of events $\mathcal{B}(\ell, \psi, t), \mathcal{A}(\ell, \psi, t)$ and the utilization value $x(\ell, \psi)$ for all the link-channel pairs, respectively. For each given topology, we run the simulation three times with different random seeds and report the result with the lowest worst case ratio for event $\mathcal{B}(\ell, \psi, t)$.

\section{B. Validation of the Developed Bounds}

1) Node-exclusive Interference Model: In the nodeexclusive interference model, only links that share a common radio or node (in the original network $G$ ) interfere with each other. Table II summarizes the simulation results for this interference model. In this table, the first column shows the topology of simulated networks, including both regular grids and random networks. G- $n$ stands for regular grid with $n$ nodes and R- $n$ stands for random network with $n$ nodes. The first parameter in the parenthesis is the percentage of idle linkchannel pairs. In one of our randomly generated networks with 100 nodes, there are still about 650 active link-channel pairs even if the idle probability is 0.95 . The second parameter in the parenthesis is the number of time slots for each run of the simulation. Since the utilization value for some link-channel pair may be low, we need to run the simulation for a large enough number of time slots to guarantee that the final result is both reasonable and valid. The second, third and fourth columns are the range ( $\min$ and $\max$ values) and average value of the sum in the LHS of (9) for all the link-channel pairs. As we can see from the third column $\max$, all the maximal sums are very close to $1 / e$. The last two columns are the worst case ratios between the probabilities of events $\mathcal{B}(\ell, \psi, t), \mathcal{A}(\ell, \psi, t)$ and the utilization value for all the linkchannel pairs, respectively. The results from Table II show that the probabilities of events $\mathcal{B}(\ell, \psi, t)$ and $\mathcal{A}(\ell, \psi, t)$ for all the link-channel pairs are larger than their utilization values $x(\ell, \psi)$ under this interference model, which demonstrate that the proposed randomized scheduling algorithm is feasible in SDR networks and our developed bounds are valid.

2) Protocol Model: Table III shows the simulation results for the protocol model. Compared with Table II, we change the 


\begin{tabular}{c|c|c|c|c|c} 
Topology & $\min$ & $\max$ & avg. & $\mathcal{B}$ & $\mathcal{A}$ \\
\hline G-16 $\left(60,1 \times 10^{5}\right)$ & 0.17 & 0.3673 & 0.27 & 1.02 & 1.22 \\
G-16 $\left(80,1 \times 10^{5}\right)$ & 0.09 & 0.3605 & 0.23 & 1.08 & 1.31 \\
G-25 $\left(80,1 \times 10^{5}\right)$ & 0.13 & 0.3675 & 0.25 & 1.04 & 1.26 \\
G-25 $\left(90,1 \times 10^{5}\right)$ & 0.03 & 0.3661 & 0.19 & 1.03 & 1.11 \\
R-20 $\left(80,1 \times 10^{5}\right)$ & 0.09 & 0.3635 & 0.25 & 1.06 & 1.16 \\
R-20 $\left(90,1 \times 10^{5}\right)$ & 0.04 & 0.3668 & 0.17 & 1.11 & 1.11 \\
R-50 $\left(90,1 \times 10^{5}\right)$ & 0.02 & 0.3671 & 0.19 & 1.01 & 1.27 \\
R-50 $\left(95,1 \times 10^{5}\right)$ & 0.02 & 0.3517 & 0.19 & 1.14 & 1.25 \\
R-100 $\left(95,2 \times 10^{5}\right)$ & 0.01 & 0.3661 & 0.19 & 1.03 & 1.21 \\
R-100 $\left(97,1 \times 10^{5}\right)$ & 0.01 & 0.3663 & 0.17 & 1.08 & 1.17
\end{tabular}

Table II

SIMULATION RESULTS FOR NODE-EXCLUSIVE INTERFERENCE MODEL.

\begin{tabular}{c|c|c|c|c|c} 
Topology & min & max & avg. & $\mathcal{B}$ & $\mathcal{A}$ \\
\hline G-16 $\left(0.5,1 \times 10^{5}\right)$ & 0.09 & 0.3674 & 0.23 & 1.06 & 1.23 \\
G-16 $\left(1.0,1 \times 10^{5}\right)$ & 0.12 & 0.3665 & 0.26 & 1.04 & 1.13 \\
G-25 $\left(0.5,1 \times 10^{5}\right)$ & 0.04 & 0.3616 & 0.21 & 1.11 & 1.19 \\
G-25 $\left(1.0,1 \times 10^{5}\right)$ & 0.09 & 0.3589 & 0.22 & 1.04 & 1.12 \\
R-20 $\left(0.5,1 \times 10^{5}\right)$ & 0.07 & 0.3665 & 0.20 & 1.14 & 1.19 \\
R-20 $\left(1.0,1 \times 10^{5}\right)$ & 0.02 & 0.3654 & 0.21 & 1.05 & 1.05 \\
R-20 $\left(1.5,1 \times 10^{5}\right)$ & 0.05 & 0.3573 & 0.25 & 1.14 & 1.20 \\
R-50 $\left(0.5,1 \times 10^{5}\right)$ & 0.01 & 0.3597 & 0.20 & 1.07 & 1.09 \\
R-50 $\left(1.0,1 \times 10^{5}\right)$ & 0.02 & 0.3658 & 0.22 & 1.01 & 1.03 \\
R-50 $\left(1.5,1 \times 10^{5}\right)$ & 0.03 & 0.3603 & 0.22 & 1.08 & 1.15 \\
R-100 $\left(0.5,2 \times 10^{5}\right)$ & 0.01 & 0.3665 & 0.20 & 1.02 & 1.09 \\
R-100 $\left(1.0,3 \times 10^{5}\right)$ & 0.05 & 0.3607 & 0.22 & 1.02 & 1.06 \\
R-100 $\left(1.5,4 \times 10^{5}\right)$ & 0.06 & 0.3675 & 0.24 & 1.01 & 1.04 \\
& & & & &
\end{tabular}

Table III

SIMULATION RESULTS FOR PROTOCOL INTERFERENCE MODEL.

first parameter in the parenthesis of the first column to be $\eta$. In this interference model, basically the value of $\eta$ determines the interference set of each link. For example, in the regular grids, when $\eta=0.5$ the receiving radio $\rho$ of a link $\ell$ will generally be interfered by radios on 7 other nodes and when $\eta=1, \rho$ will be interfered by radios on 11 other nodes. For random networks, the performance of our scheduling algorithm is evaluated with $\eta=0.5,1$ and 1.5. As $\eta$ increases, the number of links in the interference set of each link also increases, which may make the link utilization value small. So, we also increase the number of time slots for the simulations with large $\eta$. The link-channel pair idle probabilities for $4 \times 4$ and $5 \times 5$ grids are 0.8 and 0.9 , respectively. The link-channel pair idle probabilities for random networks with 20,50 and 100 nodes are $0.9,0.95$ and 0.97 , respectively. Again, all worstcase ratios between the probability of event $\mathcal{B}(\ell, \psi, t)$ and the utilization value for all the link-channel pairs are larger than 1.

3) fPrIM Interference Model: Table IV presents the simulation results for PPrIM interference model. Compared with Table II, we change the first parameter in the parenthesis of the first column to be $q$. Like the parameter $\eta$ in protocol model, the value of $q$ in PPrIM model determines the interference range of each radio. Since in this model, nodes may have nonuniform transmission ranges and interference ranges, we only conduct performance evaluation on random networks with $q=$ $0.5,1$ and 1.5. Again, the link-channel pair idle probabilities for random networks with 20,50 and 100 nodes are 0.9, 0.95

\begin{tabular}{c|c|c|c|c|c} 
Topology & min & max & avg. & $\mathcal{B}$ & $\mathcal{A}$ \\
\hline R-20 $\left(0.5,1 \times 10^{5}\right)$ & 0.03 & 0.3607 & 0.18 & 1.23 & 1.39 \\
R-20 $\left(1.0,1 \times 10^{5}\right)$ & 0.03 & 0.3677 & 0.18 & 1.20 & 1.22 \\
R-20 $\left(1.5,1 \times 10^{5}\right)$ & 0.04 & 0.3516 & 0.22 & 1.10 & 1.27 \\
R-50 $\left(0.5,2 \times 10^{5}\right)$ & 0.03 & 0.3677 & 0.22 & 1.05 & 1.13 \\
R-50 $\left(1.0,2 \times 10^{5}\right)$ & 0.09 & 0.3559 & 0.21 & 1.08 & 1.15 \\
R-50 $\left(1.5,3 \times 10^{5}\right)$ & 0.06 & 0.3552 & 0.22 & 1.00 & 1.18 \\
R-100 $\left(0.5,3 \times 10^{5}\right)$ & 0.03 & 0.3614 & 0.17 & 1.26 & 1.28 \\
R-100 $\left(1.0,3 \times 10^{5}\right)$ & 0.02 & 0.3670 & 0.18 & 1.16 & 1.18 \\
R-100 $\left(1.5,3 \times 10^{5}\right)$ & 0.02 & 0.3650 & 0.20 & 1.22 & 1.23
\end{tabular}

Table IV

SIMULATION RESULTS FOR FPRIM INTERFERENCE MODEL.

\begin{tabular}{c|c|c|c|c|c} 
Topology & $\min$ & $\max$ & avg. & $\mathcal{B}$ & $\mathcal{A}$ \\
\hline $2,5,0.95,2 \times 10^{5}$ & 0.05 & 0.3660 & 0.22 & 1.02 & 1.11 \\
$2,8,0.95,2 \times 10^{5}$ & 0.05 & 0.3643 & 0.23 & 1.01 & 1.13 \\
$3,5,0.96,3 \times 10^{5}$ & 0.05 & 0.3604 & 0.22 & 1.02 & 1.09 \\
$3,8,0.97,3 \times 10^{5}$ & 0.05 & 0.3651 & 0.21 & 1.02 & 1.09 \\
$4,5,0.98,4 \times 10^{5}$ & 0.07 & 0.3531 & 0.22 & 1.00 & 1.05 \\
$4,8,0.98,4 \times 10^{5}$ & 0.05 & 0.3669 & 0.21 & 1.05 & 1.10 \\
$5,5,0.99,5 \times 10^{5}$ & 0.04 & 0.3629 & 0.21 & 1.01 & 1.03 \\
$5,8,0.99,5 \times 10^{5}$ & 0.04 & 0.3639 & 0.21 & 1.04 & 1.10
\end{tabular}

Table V

SIMULATION RESULTS FOR SDR NETWORKS WITH MORE RADIOS AND CHANNELS.

and 0.99 , respectively. The results from Table IV demonstrate that our randomized scheduling algorithm works well under the fPrIM model. Note that we also run the simulations on other random networks for these three interference models and got similar results which are omitted due to space limitation.

4) SDR Networks with More Radios and Channels: Table V summarizes the simulation results for SDR networks with 2, 3,4 and 5 radios and with 5 and 8 channels, respectively. The results are for an example network with 100 nodes, using fPrIM interference model $(q=1.0)$. For the first column, its first parameter is the number of radios; the second parameter is the number of channels; the third parameter is the percentage of idle link-channel pairs; and the last parameter is the number of time slots for each run of the simulation. The results from Table $\mathrm{V}$ show that the worst case ratios between the probability of event $\mathcal{B}(\ell, \psi, t)$ and the utilization value are larger than 1 for all these combinations of the simulation parameters.

\section{Comparison Between the Distributed Algorithms}

To compare the performance of the PLDS and CFDS algorithms, Figure 2 and Figure 3 show the cumulative distribution function (CDF) for the ratio of successful transmissions and collisions for all the active link-channel pairs, respectively. The results are for an example network with 100 nodes, using PPrIM interference model $(q=1.5)$. The number of time slots for this simulation is 30,000 . CFDS v1 and CFDS v2 are two variances of the CFDS algorithm. For the first one, when radio $\rho$ makes transmission decision for link $\ell$, it only considers the decisions made by other transmitter radios of the links in $\ell$ 's primary interference set. For the second one, $\rho$ will consider the transmitter radios of the links in $\ell$ 's primary and secondary interference set. These two variances obey the inductive-scheduling technique strictly, 


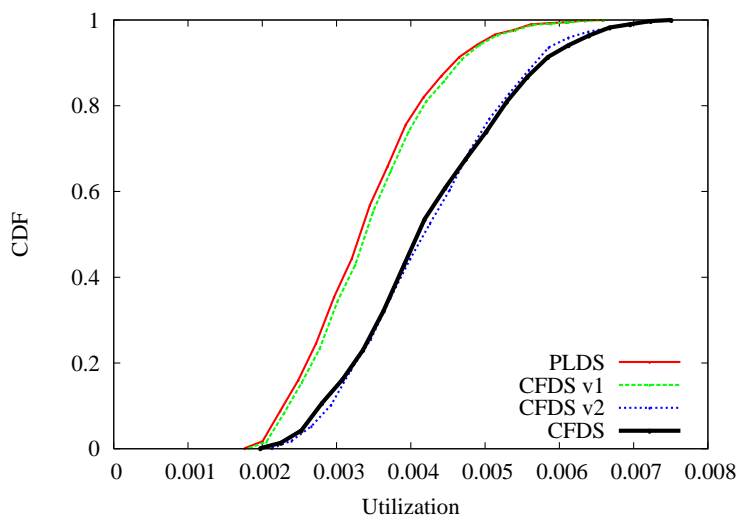

Figure 2. CDF for the ratio of successful transmissions for all the active link-channel pairs.

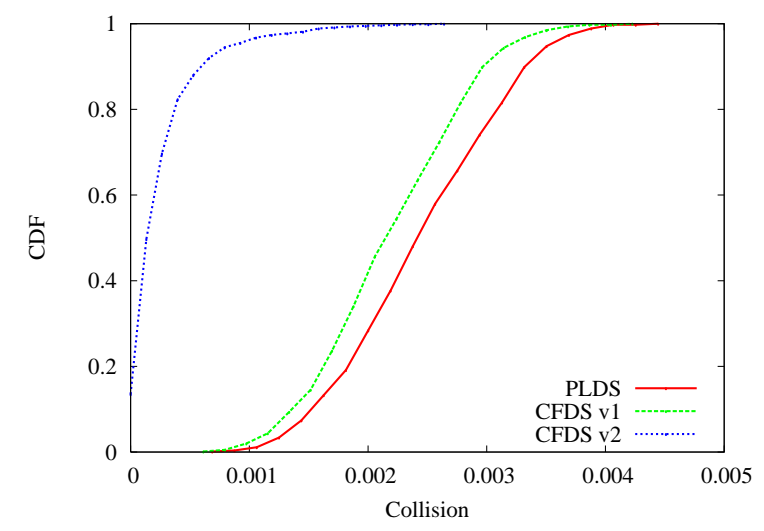

Figure 3. CDF for the ratio of collisions for active link-channel pairs.

i.e., a link will schedule its transmission if there is no links with higher inductive order schedule their transmissions. As we can see from Figure 2, compared with coordination only among primary interfering links, the coordination among both primary and secondary interfering links can improve the ratio of successful transmission. The CFDS v2 algorithm can not completely avoid collisions which is shown in Figure 3.

\section{RELATED WORK}

A large body of research decomposes the problem of crosslayer throughput maximization and treats channel assignment and link scheduling in isolation. The results of [24], [4], [21], [18], [15], [7], [16], [12] assume the availability of a scheduling protocol such as IEEE 802.11 or maximal scheduling, and focus on the design of channel assignment strategies such that the sub-network induced by each channel has good topological properties. In a similar vein, [13], [23] assume the availability of a channel-assignment protocol, and propose link-layer scheduling protocols for MCMR networks. While this modular approach has clear advantages, it is less desirable for throughput maximization:none of the above works provide an analysis of how close the throughput region achievable by their schemes are to the capacity region achievable through jointly optimal channel-assignment and link-scheduling. From this perspective, the most relevant works are [1], [8], [14], which we survey next.

Alicherry, Bhatia and Li [1] study the joint channel assignment and link scheduling problem under the uniform RTS-CTS model with parameter $q$; they proposed a centralized algorithm under the assumption that the network is homogeneous: i.e., each node has the same number of radios $\nu$, and each radio has the same set of $\kappa$ channels, and for a given link, each channel has the same capacity. For $q$ equal to 1,2 and 2.5, they prove that the throughput region yielded by their techniques is at most a factor of $\frac{4 \kappa}{\nu}, \frac{8 \kappa}{\nu}$ and $\frac{12 \kappa}{\nu}$ respectively (these are the respective competitive factors for their scheme). In contrast, our algorithmic results are derived under a generic model of interference, and the performance guarantees we present are in terms of $\lambda$ and $\Delta$, which are properties specific to a given interference model. Our algorithms and guarantees also apply for arbitrary heterogeneous networks. Most significantly, we present both centralized and distributed schemes whose guarantees improve upon those of [1]; for the special-case of the uniform RTS-CTS model studied by [1], for values of $q$ equal to 1,2 , and 2.5 respectively, our centralized scheme yields competitive factors of 6,10 , and 14 respectively, and our distributed scheme yields competitive factors of $6 e, 10 e$, and $14 e$ respectively. These factors are independent of any parameter determined by the network topology.

Kodialam and Nandagopal [8] propose two centralized heuristics - a greedy heuristic, and a packing based heuristic for the joint channel allocation and scheduling problem. Their schemes are applicable to arbitrary link-conflict based interference models. However, they do not present any guarantees for the competitiveness of their algorithms. Indeed, it is possible to construct a family of geometric network topologies where the admissible throughput region of their schemes are a factor of $\Omega(n)$ away from the optimal joint channel assignment and scheduling. Since $n$ is the number of network nodes, the competitiveness of their scheme is unbounded - even under geometric models of network interference. In contrast, we present centralized and distributed schemes with provablygood competitive guarantees; this translates to constant-factor competitiveness for several well-studied geometric models of wireless interference.

The work of Lin and Rasool [14] is most similar in spirit to our results. They present a "distributed" algorithm for joint channel assignment and link scheduling whose competitive ratio is guaranteed to be at most $\Delta+2$, where $\Delta$ is the independence number. In their scheme, each "link" in the network makes the channel assignment and scheduling decisions for each time slot by examining the instantaneous queue length of the links in its interference set during the slot. We contend that, while the scheme of Lin and Rasool is amenable to distributed implementation, it is not fully distributed in its present form. A "link" in a wireless network is a logical entity; assigning a channel to a link implies that the sender of the link decides to transmit in the channel, and the receiver simultaneously decides to listen in that channel. The packet queues for the link are maintained only at the sending end-point of the link. Thus, in order for the receiver to make channel assignment 
and scheduling decisions based on queue sizes, the sender and receiver for each network link need to exchange this information prior to each transmission. Exchanging queuesize information prior to each transmission is a significant overhead; how this information exchange is performed and how this affects the competitive ratio are critical issues that are left unaddressed in [14]. While it is in principle possible to listen in on neighbors' "current state" information in every step of the protocol, this poses challenges. First, the frequency at which a node broadcasts this information should be available at all of its neighbors, a necessary condition for which is that any pair of distance-2 nodes share a channel - a condition may not hold. Second, even if this condition holds, a node may have to listen in simultaneously on many different channels to get its neighbors' queue-state information, which may be infeasible. In contrast, observe that our protocol requires essentially no such coordination after the initial discovery of distance-two neighborhoods. The assumptions made in our distributed algorithm come with far less overhead: the endpoints of each link only need to know the long-term rate which needs to be sustained by the link as opposed to instantaneous queue sizes. In our scheme, the end-points of a link make channel assignment decisions through the use of access-hash functions. This completely eliminates the need for information exchange on a per-transmission basis, and thus makes our channel assignment and scheduling strategy truly distributed.

\section{Conclusion}

We have developed two novel provably-good (distributed) algorithms for channel allocation and scheduling in SDR networks. In the first algorithm (PLDS), each radio makes its transmission decision purely locally and does not need to exchange any information with links in its interference set. The second (CFDS) is collision-free through the use of inductivescheduling. Each radio only needs to exchange the utilization values between interfering links during the protocol setup phase; After this phase, the radios can make their decision locally for each time slot and do not need to exchange information with their interfering links any more. Simulation results show that our bounds are valid in practice and the second distributed algorithm can enable smart backoff-decisions to avoid unnecessary transmissions, which can save the valuable energy resource for nodes. We also present a centralized channel-allocation and scheduling strategy that provides the best performance ratio in comparison with all the channelassignment and scheduling strategies proposed thus far for SDR networks. We plan to investigate improvements and further applications of our access hash function methodology.

\section{ACKNOWLEDGMENT}

The authors thank Jonathan Katz for useful discussions.

\section{REFERENCES}

[1] M. Alicherry, R. Bhatia, and L. E. Li. Joint channel assignment and routing for throughput optimization in multi-radio wireless mesh networks. In MobiCom '05: Proceedings of the 11th annual international conference on Mobile computing and networking, pages 58-72, New York, NY, USA, 2005. ACM Press.
[2] B. Awerbuch and R. Khandekar. Greedy distributed optimization of multi-commodity flows. In Proc. ACM Symposium on Principles of Distributed Computing, pages 274-283, 2007.

[3] R. Bruno, M. Conti, and E. Gregori. Optimal capacity of p-persistent CSMA protocols. IEEE Commun. Lett., 7:139-141, 2003.

[4] A. Brzezinski, G. Zussman, and E. Modiano. Enabling distributed throughput maximization in wireless mesh networks: a partitioning approach. In MobiCom '06: Proceedings of the 12th annual international conference on Mobile computing and networking, pages 26-37, New York, NY, USA, 2006. ACM.

[5] N. Garg and J. Koenemann. Faster and simpler algorithms for multicommodity flow and other fractional packing problems. In Proceedings of the 39th Annual Symposium on Foundations of Computer Science, page 300. IEEE Computer Science Society, 1998.

[6] P. Gupta and P. R. Kumar. The capacity of wireless networks. IEEE Transactions on Information Theory, 46(2):388-404, 2000.

[7] B.-J. Ko, V. Misra, J. Padhye, and D. Rubenstein. Distributed channel assignment in multi-radio 802.11 mesh networks. In Wireless Coтmunications and Networking Conference, pages 3978-3983, 2007.

[8] M. Kodialam and T. Nandagopal. Characterizing the capacity region in multi-radio multi-channel wireless mesh networks. In MobiCom '05: Proceedings of the 11th annual international conference on Mobile computing and networking, pages 73-87, New York, NY, USA, 2005. ACM Press.

[9] V. S. A. Kumar, M. V. Marathe, S. Parthasarathy, and A. Srinivasan. Endto-end packet-scheduling in wireless ad-hoc networks. In SODA '04: Proceedings of the fifteenth annual ACM-SIAM symposium on Discrete algorithms, pages 1021-1030, Philadelphia, PA, USA, 2004. Society for Industrial and Applied Mathematics.

[10] V. S. A. Kumar, M. V. Marathe, S. Parthasarathy, and A. Srinivasan. Algorithmic aspects of capacity in wireless networks. In SIGMETRICS '05: Proceedings of the 2005 ACM SIGMETRICS International Conference on Measurement and Modeling of Computer Systems, pages 133-144, 2005.

[11] V. S. A. Kumar, M. V. Marathe, S. Parthasarathy, and A. Srinivasan. Provable algorithms for joint optimization of transport, routing and MAC layers in wireless ad hoc networks. In Proc. DialM-POMC Workshop on Foundations of Mobile Computing, 2007. Eight pages.

[12] P. Kyasanur and N. H. Vaidya. Routing and interface assignment in multi-channel multi-interface wireless networks. In IEEE Wireless Communications and Networking Conference, volume 4, pages 20512056, 2005.

[13] P. Kyasanur and N. H. Vaidya. Routing and link-layer protocols for multi-channel multi-interface ad hoc wireless networks. SIGMOBILE Mob. Comput. Commun. Rev., 10(1):31-43, 2006.

[14] X. Lin and S. Rasool. A distributed and provably efficient joint channelassignment, scheduling and routing algorithm for multi-channel multiradio wireless mesh networks. In Proc. of IEEE INFOCOM, 2007.

[15] M. K. Marina and S. R. Das. A topology control approach for utilizing multiple channels in multi-radio wireless mesh networks. In BROADNETS, pages 412-421, 2005.

[16] K. N. Ramachandran, E. M. Belding-Royer, K. C. Almeroth, and M. M. Buddhikot. Interference-aware channel assignment in multi-radio wireless mesh networks. In Proc. of IEEE INFOCOM, 2006.

[17] S. Ramanathan and E. L. Lloyd. Scheduling algorithms for multihop radio networks. IEEE-ACM Transactions on Networking (ToN), 1:166177, 1993.

[18] A. Raniwala, K. Gopalan, and T.-C. Chiueh. Centralized channel assignment and routing algorithms for multi-channel wireless mesh networks. SIGMOBILE Mob. Comput. Commun. Rev., 8(2):50-65, 2004.

[19] G. Sharma, R. R. Mazumdar, and N. B. Shroff. On the complexity of scheduling in wireless networks. In MobiCom '06: Proceedings of the 12th annual international conference on Mobile computing and networking, pages 227-238, New York, NY, USA, 2006. ACM Press.

[20] Y. Shi and T. Hou. Optimal power control for multi-hop software defined radio networks. In Proc. of IEEE INFOCOM, 2007.

[21] A. P. Subramanian, H. Gupta, and S. R. Das. Minimum interference channel assignment in multi-radio wireless mesh networks. In Proc. of the 4th IEEE International Conference of Sensor and Ad Hoc Communications Networks (SECON 2007), San Diego, California, USA, June 2007.

[22] W. Wang, X.-Y. Li, O. Frieder, Y. Wang, and W.-Z. Song. Efficient interference-aware TDMA link scheduling for static wireless networks. In MobiCom '06: Proceedings of the 12th annual international confer- 
ence on Mobile computing and networking, pages 262-273, New York, NY, USA, 2006. ACM Press.

[23] S.-L. Wu, C.-Y. Lin, Y.-C. Tseng, and J.-P. Sheu. A new multi-channel MAC protocol with on-demand channel assignment for multi-hop mobile ad hoc networks. In ISPAN, pages 232-237, 2000.

[24] K. Xing, X. Cheng, L. Ma, and Q. Liang. Superimposed code based channel assignment in multi-radio multi-channel wireless mesh networks. In MobiCom '07: Proceedings of the 13th annual ACM international conference on Mobile computing and networking, pages 15-26, New York, NY, USA, 2007. ACM Press.

[25] S. Yi, Y. Pei, and S. Kalyanaraman. On the capacity improvement of ad hoc wireless networks using directional antennas. In MobiHoc 2003: Proceedings of the 4th ACM International Symposium on Mobile Ad Hoc Networking and Computing, pages 108-116, 2003. 